\title{
Efficacy and Safety of a New Formulation of Ferric Sodium EDTA Associated with Vitamin C, Folic Acid, Copper Gluconate, Zinc Gluconate and Selenomethionine Administration in Patients with Secondary Anaemia
}

\author{
Annalisa Curcio ${ }^{1}$, Adriana Romano ${ }^{1}$, Marchitto Nicola ${ }^{2}$, Michele Pironti ${ }^{1 *}$ and Raimondi Gianfranco ${ }^{3}$ \\ ${ }^{1}$ Merqurio Pharma, Naples, Italy \\ ${ }^{2}$ Department of Internal Medicine, Alfredo Fiorini Hospital, Terracina, (Latina), Italy \\ ${ }^{3}$ Department of Medical-surgical Sciences and Biotechnologies, "Sapienza" University of Rome, Italy
}

*Corresponding author: Pironti M, Merqurio Pharma, Corso Umberto I, 23-80138, Naples, Italy, Tel: +39 0815524300; Fax: +39 0814201136; E-mail: michele.pironti@merqurio.it

Received date: September 7, 2018; Accepted date: September 11, 2018; Published date: September 14, 2018

Copyright: (C) 2018 Curcio A, et al. This is an open-access article distributed under the terms of the Creative Commons Attribution License, which permits unrestricted use, distribution, and reproduction in any medium, provided the original author and source are credited.

\begin{abstract}
Anemia is a global problem since two billion people are affected by blood cells disorders. Anemia may reduce the quality of life of affected patients and may also to get worse the outcome and quality of life of patients with comorbidities as kidney failure, heart failure, arrhythmia, coronary heart disease and so on. In patients with coronary heart disease, anginal episodes may increase in frequency and severity, and patients with kidney failure may have an increased number of re-hospitalizations.
\end{abstract}

Here we report the effectiveness of the therapy with Ferric Sodium EDTA, in combination with vitamin C, folic acid, copper gluconate, zinc gluconate and selenomethionine (FERACHEL FORTE ${ }^{\circledR}$ ) that has shown several advantages in daily clinical practice.

Keywords: Anaemia; Ferric sodium EDTA; Cardiovascular risk

\section{Background}

Anemia is a global problem since two billion people are affected by blood cells disorders.

According to WHO criteria, anemia is defined as blood hemoglobin $(\mathrm{Hb})$ concentration $<13 \mathrm{~g} / \mathrm{dL}$ or hematocrit $(\mathrm{Hct})<39 \%$ in adult males or $\mathrm{Hb}$ concentration $<12 \mathrm{~g} / \mathrm{dL}$ or $\mathrm{Hct}<37 \%$ in adult females.

Iron-deficiency anemia is the most common type of anemia in young adults in particular in premenopausal women. Iron-deficiency anemia is divided in three different clinical forms from a pathophysiological point of view: increased iron requirement, increased iron loss, reduced iron intake from diet or due to gastrointestinal abnormalities. Furthermore, in daily clinical practice, the most common cause of iron deficiency anemia is clinically related to increased blood loss as metrorrhagia or gastrointestinal bleeding [1].

Iron deficiency anemia can be also linked to a chronic disease as kidney failure, heart failure, coronary heart disease. The anaemia may induce a worsening of clinical conditions that need to be improved [2].

There are two different types of iron supplements: ferric iron supplements (containing $\mathrm{Fe}^{3+}$ ) and ferrous iron supplements (containing $\mathrm{Fe}^{2+}$ ) [3-4].

An important source of ferric iron $\left(\mathrm{Fe}^{3+}\right)$ is Ferric Sodium EDTA which consists in $\mathrm{Fe} 3+$ chelated with ethylendiamminotetraacetic acid (EDTA).
The iron from ferric sodium EDTA is highly soluble, as it remains bound to EDTA in the acid milieu of the stomach, and becomes released only in the more alkaline medium of the duodenum and of the small intestine, where it is subsequently absorbed in the bloodstream.

Ferric sodium EDTA represents a new iron source in order to treat iron deficiency with interesting features: it is taste-less, it does not interact with foods, it is completely water soluble, and without metallic taste; furthermore, it does not cause teeth and stools staining (especially relevant in case of gastrointestinal diagnostic investigations) and its absorption is complete also in presence of inhibitors of iron absorption as phytates [5].

In last years literature offered many evidences about the advantages of Ferric Sodium EDTA, in particular if it is associated with other components and trace elements as folic acid, vitamin C, copper, zinc and selenium and here we report all clinical improvements obtained with this iron source administered orally (FERACHEL FORTE ${ }^{\circ}$ ) in different clinical conditions in which it has been tested.

\section{Secondary Iron-deficiency Anemia}

A multicenter and observational registry enrolled patients with different stages of iron-deficiency anaemia. The study evaluated the effects of the oral Ferric sodium EDTA associated with folic acid, vitamin $\mathrm{C}$, copper, zinc and selenium (FERACHEL FORTE ${ }^{\circ}$ ) on blood parameters and on clinical outcomes (unpublished data).

The study enrolled 111 patients with iron-deficiency anemia: 31 patients with mild iron deficiency anaemia, 62 patients with moderate iron deficiency anaemia and 18 patients with severe iron-deficiency 
anemia. All patients were treated with 1 tablet a day of Ferric sodium EDTA for 72 days. The patients were evaluated at three steps: a) time 0 (before the treatment), b) time 1 (after 24 days of treatment) and c) time 2 (after 72 days of treatment) (unpublished data).

Blood parameters, clinical symptoms and side effects were recorded in all steps of the study.

A progressive increase of iron in the blood was recorded at time 1 and 2 of the study with a respective increase of $13 \mathrm{mcg} \backslash \mathrm{dL}$ and $33 \mathrm{mcg}$ $\backslash \mathrm{dL}$ in the ferritin levels; also haemoglobin levels increased of 1,2 $(\mathrm{g} / \mathrm{dL}) \mathrm{Hb}$ at time 1 and 2,2 (g/dL) Hb at time 2 (unpublished data).

Nearly $78 \%$ of patients at time 2 reported a reduction of clinical symptoms associated with iron deficiency and its related anemia; in particular the treated patients referred a decrease of symptoms as tiredness, fatigue, weakness, andtachycardia.

The $96 \%$ of patients did not refer side effects during the treatment with Ferric sodium EDTA in association (FERACHEL FORTE). The remaining $4 \%$ of patients referred aspecific symptoms as side effects that could be related also to their further chronic pathologies (unpublished data).

\section{Cardiovascular Diseases and Iron-deficiency Anemia}

Several physicians recently underlined that administration of oral ferric iron $(\mathrm{Fe} 3+)$ is associated with improvements of cardiovascular outcomes and quality of life [6].

Anemia is a common comorbidity of chronic cardiovascular disease as heart failure, chronic coronary artery disease, atrial fibrillation [7-9]. It is a common disorder of the elderly and its prevalence increases with age. This can be related to several causes as the reduced production of $\mathrm{HB}$ by bone marrow, the reduced intake of microelements as iron, folic acid and vitamin B12, or intestinal microloss for the chronic use of antithrombotic drugs (antiplatelets or anticoagulants).

In a specific study the effect of Ferric Sodium EDTA $60 \mathrm{mg}(2$ tabs/24 h) associated with folic acid, vitamin C, copper, zinc and selenium (FERACHEL FORTE ${ }^{\circ}$ ) in elderly patients with secondary anemia has been tested vs. intravenous polysaccharide $\mathrm{Fe}++$ in patients with chronic cardiovascular diseases associated to iron deficiency anemia [10].

The therapy with Ferric SodiumEDTA, in combination with vitamin $\mathrm{C}$, folic acid, copper gluconate, zinc gluconate and selenomethionine (FERACHEL FORTE ${ }^{\circ}$ ) was noted to have a real superiority in comparison with the intravenous administration of polysaccharide $\mathrm{Fe}+$ + about arrhythmic risk and quality of life of several patients with chronic cardiovascular disease. The safety of this therapeutic regimen was confirmed also by the percentage of side effects.

\section{Conclusion}

In last years the good clinical practice of chronic diseases in adults and in elderly patients underlined an increased number of patients affected by secondary anemia. However, iron deficiency anemia remains the most common type of secondary anemia in these clinical settings.

A common trouble of oral iron-based food supplements is the significant number of side effects on gastrointestinal functions in addition to the time of treatment of about three months with the first signs of effectiveness after at least 40 day of treatment.

The therapy with a new oral source of iron (i.e., Ferric Sodium EDTA, in combination with vitamin $\mathrm{C}$, folic acid, copper gluconate, zinc gluconate and selenomethionine, FERACHEL FORTE ${ }^{*}$ ) revealed a good effectiveness in blood iron increase and a significant safety with a reduced percentage of side effects in several chronic diseases showing efficacy evidence already after 24 days of treatment allowing a cycle therapy of about 72 days compared to standard therapies.

\section{References}

1. Polin V, Coriat R, Perkins G, Dhooge M, Abitbol V, et al. (2013) Iron deficiency: from diagnosis to treatment. Dig Liver Dis 45: 803-809.

2. Motonishi S, Tanaka K, Ozawa T (2018) Iron deficiency associates with deterioration in several symptoms independently from hemoglobin level among chronic hemodialysis patients. PLoS One 13: e0201662.

3. Lichtenstein GR, Onken JE (2018) Improved Hemoglobin Response with Ferric Carboxymaltose in Patients with Gastrointestinal-Related IronDeficiency Anemia Versus Oral Iron. Dig Dis Sci.

4. Ikuta K, Hanashi H, Hirai K, Ota Y, Matsuyama Y, et al. (2018) Comparison of efficacy and safety between intravenous ferric carboxymaltose and saccharated ferric oxide in Japanese patients with iron-deficiency anemia due to hypermenorrhea: a multi-center, randomized, open-label noninferiority study. Int J Hematol.

5. Cignini P, Mangiafico L, Padula F, D'Emidio L, Dugo N, et al. (2015) Supplementation with a dietary multicomponent $\left(\right.$ Lafergin $\left.\left({ }^{\circ}\right)\right)$ based on Ferric Sodium EDTA (Ferrazone( 9 ): results of an observational study. J Prenat Med 9: 1-7.

6. Kang CK, Pope M, Lang CC, Kalra PR (2017) Iron deficiency in heart failure: Efficacy and safety of intravenous iron therapy. Cardiovasc Ther 35.

7. Zeller T, Waldeyer C, Ojeda F, Schnabel RB, Schäfer S, et al. (2018) Adverse Outcome Prediction of Iron Deficiency in Patients with Acute Coronary Syndrome. Biomolecules 8: 60 .

8. Bekfani T, Pellicori P, Morris D, Ebner N, Valentova M, et al. (2018) Iron deficiency in patients with heart failure with preserved ejection fraction and its association with reduced exercise capacity, muscle strength and quality of life. Clin Res Cardiol.

9. Pope M, Kalra PR (2018) Iron Deficiency in Heart Failure: to Treat or Not to Treat? Curr Treat Options Cardiovasc Med 20: 65.

10. Marchitto N, Sindona F, Pannozzi A, Petrucci A, Fusco L, et al. (2018) Role of Ferric Sodium EDTA associated with vitamin C, folic acid, copper gluconate, zinc gluconate and selenomethionine administration in patients with secondary anaemia. Effects on hemoglobin value and cardiovascular risk. Transfusion Medine 2018, in press. 